\title{
Hemosiderin-laden macrophages are an independent factor correlated with pulmonary vascular resistance in idiopathic pulmonary fibrosis: a case control study
}

Jun Fukihara ${ }^{1}$, Hiroyuki Taniguchi ${ }^{1 *}$, Masahiko Ando², Yasuhiro Kondoh¹, Tomoki Kimura', Kensuke Kataoka', Taiki Furukawa', Takeshi Johkoh ${ }^{3}$, Junya Fukuoka ${ }^{4}$, Koji Sakamoto $^{5}$ and Yoshinori Hasegawa ${ }^{5}$

\begin{abstract}
Background: Increases in hemosiderin-laden macrophages (HLM) are reported to be observed in idiopathic pulmonary fibrosis (IPF). According to a recent study, significant correlation between hemosiderin deposition in the lung tissue of IPF and pulmonary hypertension evaluated by echocardiography has been suspected. In this study, we aimed to evaluate whether HLM in bronchoalveolar lavage fluid (BALF) is a factor correlated with pulmonary hemodynamic parameters evaluated by right heart catheterization in patients with IPF.

Methods: Initial data from 103 consecutive patients with IPF who underwent surgical lung biopsy between November 2007 and March 2014 were retrospectively analyzed. The "HLM score" of BALF was established by dividing the number of Perls' Prussian blue stain positive macrophages by the total number of macrophages counted.

Results: BALF showed an elevated HLM score (38.2\%). Right heart catheterization revealed mean pulmonary arterial pressure (mPAP) of $16.3 \mathrm{mmHg}$ and pulmonary vascular resistance (PVR) of 1.55 Wood units. HLM score was positively correlated with mPAP $(\rho=0.204 ; p=0.038)$ and PVR $(\rho=0.349, p<0.001)$. In multivariate analysis, 6 -min walk distance (standardized partial regression coefficient $[\beta],-0.391 ; p<0.001$ ), minimum oxygen saturation during 6 -min walk distance $(\beta,-0.294 ; p=0.001)$ and HLM score $(\beta, 0.265 ; p=0.002)$ were independently correlated with PVR.
\end{abstract}

Conclusions: HLM score in BALF is an independent factor correlated with PVR in patients with IPF.

Keywords: Hemosiderin-laden macrophages, Idiopathic pulmonary fibrosis, Pulmonary vascular resistance, Mean pulmonary arterial pressure, Bronchoalveolar-lavage, Right heart catheterization

\section{Background}

Hemosiderin-laden macrophages (HLM) in bronchoalveolar lavage fluid (BALF) were originally known as a diagnostic biomarker of alveolar hemorrhage [1, 2]. Recently, hemosiderin deposition or exaggerated numbers of HLM have also been observed in idiopathic pulmonary fibrosis (IPF) [3, 4], which is a progressive interstitial lung disease characterized by a poor prognosis, limited response to treatment and a histopathological pattern of usual interstitial pneumonia (UIP) [5]. In

\footnotetext{
* Correspondence: taniguchi@tosei.or.jp

'Department of Respiratory Medicine and Allergy, Tosei General Hospital, 160 Nishioiwake-cho, Seto-shi, Aichi 489-8642, Japan

Full list of author information is available at the end of the article
}

these reports, a relationship between hemosiderin deposition and elevated pulmonary arterial pressure was suspected [3, 4].

In 2010, Kim et al. reported that iron deposition in the lung tissue of IPF is significantly correlated with elevated right ventricular systolic pressure (RVSP) measured by echocardiography [3]. Puxeddu et al. later demonstrated elevated HLM in the BALF of patients with IPF, especially of severer patients and patients with higher RVSP [4]. However, whether HLM in the BALF of patients with IPF is correlated with parameters measured by right heart catheterization (RHC) has never been clarified.

Among parameters measured by RHC, several recent studies have reported that pulmonary vascular resistance 
(PVR) is the strongest prognostic factor of interstitial lung diseases [6,7]. While there are some reports on predictive factors for mean pulmonary arterial pressure (mPAP) [8-12], such as diffusing capacity of the lung for carbon monoxide $\left(\mathrm{D}_{\mathrm{L}} \mathrm{CO}\right)$ [8-10], 6-min walk distance (6MWD) [11, 12], minimum oxygen saturation during 6-min walk test $\left(\mathrm{min}-\mathrm{SpO}_{2}\right)[9,11]$ and others, the correlation between PVR and other clinical variables has not been well discussed.

The aim of this retrospective study was to evaluate whether HLM in BALF is correlated with PVR in patients with IPF, together with other possible correlating factors measured at initial evaluation, such as pulmonary function, oxygen saturation and 6-min walk test measurements.

\section{Methods}

\section{Study population}

This study was approved by Institutional Review Board of Tosei General Hospital (Review Board No. 480, Seto, Aichi, Japan). Informed consent has not been obtained from participants because this is a retrospective study and the data were analyzed anonymously. A retrospective review of the initial evaluation data from 310 consecutive patients with interstitial lung disease who underwent surgical lung biopsy at Tosei General Hospital between November 2007 and March 2014 was undertaken. After excluding 94 cases with connective tissue diseases (patients fulfilling the American College of Rheumatology criteria for rheumatoid arthritis [13], systemic lupus erythematosus $[14,15]$, systemic sclerosis [16], Sjögren's syndrome [17], polymyositis-dermatomyositis [18], or mixed connective tissue disease [19]), hypersensitivity pneumonitis, vasculitis or other known causes, 216 patients were diagnosed with idiopathic interstitial pneumonia. From 2011 onward, multidisciplinary diagnoses were made after close communication between clinicians, radiologists and pathologists, and 105 patients with IPF were detected according to the guidelines for IPF [5]. After excluding one patient whom HLM in BALF could not be evaluated due to insufficient total cell count and one who did not take RHC, a total of 103 patients were finally enrolled in this study (Fig. 1). Pulmonary arterial wedge pressure of all of 103 patients was $\leq 15 \mathrm{mmHg}$. After conducting a clinical workup, we performed bronchoalveolar lavage (BAL) and $\mathrm{RHC}$ as a part of the initial evaluation before surgical lung biopsy. Characteristics of patients before BAL, including demographics, detailed clinical history, results of pulmonary function tests, serologic tests, arterial blood gas analysis and six-minute walk tests, were collected from their clinical charts.

\section{Pulmonary function tests}

Pulmonary function tests performed before BAL (within 1 month) were used for analysis. Forced vital capacity

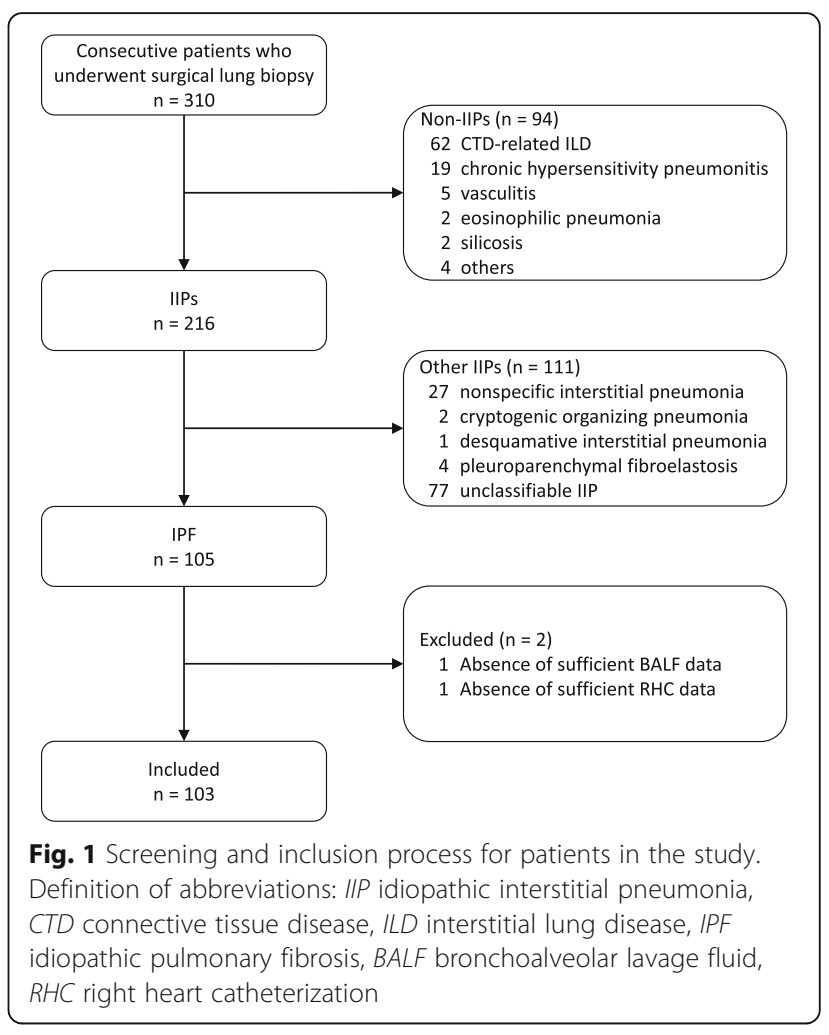

and $\mathrm{D}_{\mathrm{L}} \mathrm{CO}$ were measured by means of CHESTAC-55 V (Chest, Tokyo, Japan). Pulmonary function was measured according to the American Thoracic Society/European Respiratory Society recommendation as a physiological assessment $[20,21]$. The results were expressed as percentages of the normal predicted values.

\section{High resolution computed tomography}

High resolution computed tomography was performed with 0.5 - $\mathrm{mm}$ thick sections. An expert thoracic radiologist with 26 years of experience who was blinded to the clinical information or histological diagnosis reviewed the scans. The probability of usual interstitial pneumonia was evaluated and categorized according to the guidelines for IPF [5].

\section{Six-minute walk test}

Six-minute walk test was conducted according to the American Thoracic Society statement [22]. Briefly, all patients were tested under standardized conditions by trained technicians. Patients were instructed to walk as far as possible in $6 \mathrm{~min}$. The distance that patients could walk was recorded as 6MWD. Oxygen saturation was also measured by pulse oximetry during the test and $\mathrm{min}-\mathrm{SpO}_{2}$ was recorded. Patients underwent the tests twice to minimize the training effects. 


\section{Bronchoalveolar lavage and detection of hemosiderin laden macrophages}

Flexible bronchoscopy was performed and processed following a method described in the literature [23]. A flexible bronchoscope (BF-1 T260; Olympus Optical Co., Tokyo, Japan) was wedged into a segmental bronchus of the middle lobe or the lingula. Sequential infusions of $50 \mathrm{~mL}$ sterile normal saline were instilled three times, then immediately aspirated by manual suction after each instillation through the bronchoscope. The total volume of recovered fluid was measured, and a sample of the fluid was used for bacteriological and fungal studies. The remaining fluid was filtered through a double layer of surgical gauze and used for cell counts and cytological examination.

The cell pellets were separated from supernatant by low-speed centrifugation at $300 \times \mathrm{g}$ for $5 \mathrm{~min}$ at $4{ }^{\circ} \mathrm{C}$ (CytoSpin; Thermo Fischer Scientific Inc., Waltham, MA). Differential cell counts were made from a total count of $\geq 300$ cells stained by Diff-Quick ${ }^{\mathrm{mm}}$ stain (Scientific Products, McGraw Park, IL). HLM were detected by Perls' Prussian blue stain and counted according to the procedure of De Lassence et al. [24]. Two hundred alveolar macrophages were examined at a magnification of $\times 500$. The "HLM score" was established by dividing the number of Perls' Prussian blue stain-positive macrophages by the total number of macrophages counted.

\section{Right heart catheterization}

RHC was performed percutaneously using a Swan-Ganz catheter via either the cubital vein or the femoral vein. Cardiac output and cardiac index were calculated by the thermodilution method. PVR was calculated using the formula: $\mathrm{PVR}=(\mathrm{mPAP}-$ pulmonary arterial wedge pressure)/cardiac output.

\section{Surgical lung biopsy and pathological diagnosis}

Surgical lung biopsies were performed by either open lung biopsy or video-assisted thoracoscopic surgery. Formalinfixed, paraffin-embedded tissue was used for this study. For proof of histology, all available lung biopsy specimens were reviewed by a pulmonary pathologist with 20 years of experience who was blinded to clinical and radiological information.

\section{Statistical analysis}

Continuous variables were presented as mean \pm standard deviation or median (range), as appropriate. Categorical variables were summarized by frequency and percentage.

Univariate relationships between MPAP or PVR and other variables were evaluated using Spearman rank correlation test for continuous variables or Student's $t$ test for categorical variables, since PVR had a normal distribution (data not shown). To avoid multicollinearity, only one of the highly correlated variables (correlation coefficient $\geq 0.7$ ) was entered in the multivariate model predicting PVR, if present. The stepwise linear regression analysis model was constructed to identify independent predictors of PVR. All statistical tests were two sided, and values of $<0.05$ were considered statistically significant. Statistical analyses were carried out using SPSS version 21.0 (SPSS Inc., Chicago, IL).

\section{Results}

\section{Patient characteristics}

All the initial evaluation exams, including pulmonary function test, high resolution computed tomography, six-minute walk test, BAL and RHC, of the 103 patients enrolled in this study were performed in a median time of 22 days (range 2-115). Their clinical characteristics (Table 1) showed a predominance of males and patients with smoking history. Median age was 66 years old. Baseline predicted value for forced vital capacity and $\mathrm{D}_{\mathrm{L}} \mathrm{CO}$ were $86.8 \pm$ $20.3 \%$ and $63.7 \pm 20.3 \%$ of normal predicted value, respectively. Partial pressure of oxygen in arterial blood was $82.4 \pm 11.4$ Torr in room air. 6MWD was $608 \pm 140 \mathrm{~m}$ with min- $-\mathrm{SpO}_{2}$ of $85.0 \pm 7.6 \%$. BALF showed almost normal differential cell counts with elevated HLM score (38.2 $\pm 24.2 \%$, Table 2, Fig. 2). RHC revealed mPAP of $16.3 \pm 4.2 \mathrm{mmHg}$ and PVR of $1.55 \pm 0.81$ Wood units with normal left heart function (Table 3 ).

Table 1 Characteristics of patients before bronchoalveolar lavage

\begin{tabular}{ll}
\hline Characteristics & $N=103$ \\
\hline Age, years & $66(48 \sim 76)$ \\
Male gender & $80(77.7)$ \\
Smokers & $80(77.7)$ \\
Brinkman index & $630 \pm 581$ \\
BNP, pg/ml & $28.7 \pm 38.7$ \\
Pulmonary function test & \\
$\quad \mathrm{FVC}, \%$ pred & $86.8 \pm 20.3$ \\
$\quad \mathrm{D}_{\mathrm{L}} \mathrm{CO}, \%$ pred & $63.7 \pm 20.3$ \\
Arterial blood gas analysis & \\
$\quad$ PaO & \\
$\quad$ PaCorr & \\
Six-minute walk test & $82.4 \pm 11.4$ \\
$\quad$ Six-minute walk distance, $\mathrm{m}$ & $41.0 \pm 3.6$ \\
$\quad$ Min-SpO & \\
\hline
\end{tabular}

Values are presented as number (\%), mean \pm standard deviation or median (range). Definitions of abbreviations: BNP brain natriuretic peptide, FVC forced vital capacity, \% pred percent of normal predicted value, $D_{L} C O$ diffusing capacity of the lung for carbon monoxide, $\mathrm{PaO}_{2}$ partial pressure of oxygen in arterial blood, $\mathrm{PaCO}_{2}$ partial pressure of carbon dioxide in arterial blood, $\min -\mathrm{SpO}_{2}$ minimum oxygen saturation during 6-min walk test 
Table 2 Bronchoalveolar lavage fluid analysis

\begin{tabular}{ll}
\hline Characteristics & $N=103$ \\
\hline Total cell counts, $\times 10^{5} / \mathrm{ml}$ & $1.98 \pm 1.87$ \\
Differential cell count & \\
$\quad$ Neutrophils, \% & $0.3(0.0 \sim 63.6)$ \\
Lymphocytes, \% & $2.5(0.0 \sim 36.8)$ \\
Eosinophils, \% & $0.2(0.0 \sim 35.0)$ \\
Macrophages, \% & $94.0(34.8 \sim 99.8)$ \\
HLM score, \% & $38.2 \pm 24.2$
\end{tabular}

Values are presented as mean \pm standard deviation or median (range).

Definition of abbreviation: HLM hemosiderin-laden macrophage

\section{Correlation between MPAP, PVR and HLM scores}

HLM score showed significant positive correlations with $\operatorname{mPAP}(\rho=0.204 ; p=0.038)$ and PVR $(\rho=0.349, p<0.001)$. Due to the clinical significance of PVR compared with mPAP as a prognostic factor for interstitial lung diseases $[6,7]$, PVR was selected for use in the following analysis in this study.

\section{Factors correlated with PVR}

In the univariate analysis, partial pressure of oxygen in arterial blood, $\mathrm{D}_{\mathrm{L}} \mathrm{CO}, 6 \mathrm{MWD}$ and $\mathrm{min}-\mathrm{SpO}_{2}$ were negatively correlated with PVR, while age and HLM score

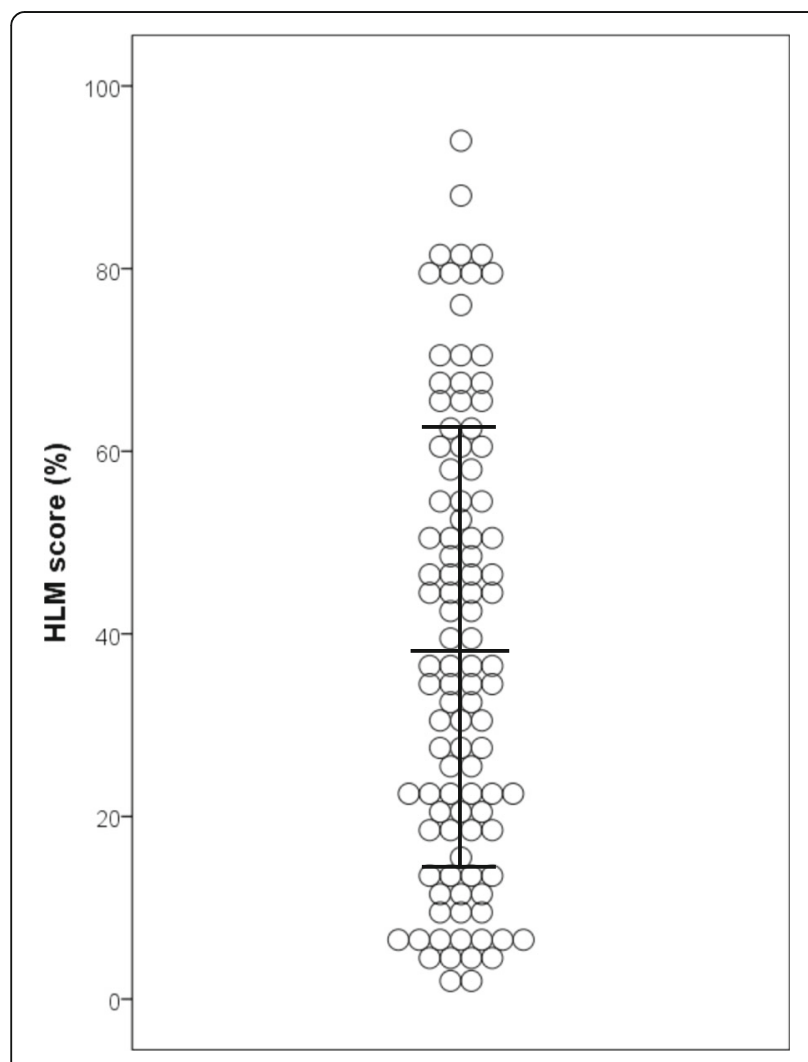

Fig. 2 Scatter plot of HLM score. Data is shown as mean \pm SD. Definition of abbreviation: HLM hemosiderin-laden macrophages
Table 3 Pulmonary arterial catheterization analysis

\begin{tabular}{lc}
\hline Characteristics & $N=103$ \\
\hline mPAP, mmHg & $16.3 \pm 4.2$ \\
PVR, Wood units & $1.55 \pm 0.81$ \\
PVRI, Wood units $\times \mathrm{m}^{2}$ & $2.66 \pm 1.32$ \\
PAWP, $\mathrm{mmHg}$ & $7.8 \pm 3.3$ \\
$\mathrm{Cl}, \mathrm{L} / \mathrm{min} / \mathrm{m}^{2}$ & $3.1 \pm 0.5$
\end{tabular}

Values are presented as mean \pm standard deviation. Definitions of abbreviations: mPAP mean pulmonary arterial pressure, PVR pulmonary vascular resistance, $P V R I$ pulmonary vascular resistance index, $P A W P$ pulmonary arterial wedge pressure, $\mathrm{Cl}$ cardiac index

were positively correlated (Table 4). No significant multicollinearity was found between the variables shown in table 4 . In the stepwise linear regression analysis including all of the variables shown in table 4, 6MWD (standardized partial regression coefficient $[\beta],-0.391 ; 95 \%$ confidence interval [95\% CI], $-0.587 \sim-0.196 ; p<0.001)$, min- $\mathrm{SpO}_{2}$ $(\beta,-0.294 ; 95 \% \mathrm{CI},-0.455 \sim-0.133 ; p=0.001)$ and HLM score $(\beta, 0.265 ; 95 \% \mathrm{CI}, 0.088 \sim 0.412 ; p=0.002)$ were independently correlated with PVR (Table 5 ).

\section{Discussion}

This is the first report to show a significant correlation between pulmonary hemodynamic parameters measured by RHC and HLM in BALF in patients with IPF. HLM score in particular was independently correlated with

Table 4 Univariate analysis between PVR and baseline characteristics

\begin{tabular}{|c|c|c|c|}
\hline Characteristics & $t$ & $\rho$ & $P$-value \\
\hline Age & & 0.230 & 0.020 \\
\hline Gender (male) & -1.035 & & 0.305 \\
\hline Smoking history (smokers) & -1.279 & & 0.207 \\
\hline BNP & & -0.091 & 0.361 \\
\hline FVC, \% pred & & -0.124 & 0.211 \\
\hline $\mathrm{D}_{\mathrm{L}} \mathrm{CO}, \%$ pred & & -0.518 & $<0.001$ \\
\hline $\mathrm{PaO}_{2}$ & & -0.424 & $<0.001$ \\
\hline $\mathrm{PaCO}_{2}$ & & -0.167 & 0.091 \\
\hline Six-minute walk distance & & -0.583 & $<0.001$ \\
\hline $\mathrm{Min}-\mathrm{SpO}_{2}$ & & -0.318 & 0.001 \\
\hline BALF-neutrophils, \% & & 0.142 & 0.152 \\
\hline BALF-lymphocytes, \% & & 0.098 & 0.325 \\
\hline BALF-eosinophils, \% & & 0.113 & 0.255 \\
\hline HLM score & & 0.349 & $<0.001$ \\
\hline
\end{tabular}

Definitions of abbreviations: PVR pulmonary vascular resistance, $t$ Student's $t, \rho$ Spearman's correlation coefficient, BNP brain natriuretic peptide, FVC forced vital capacity, \% pred percent of normal predicted value, $D_{L} C O$ diffusing capacity of the lung for carbon monoxide, $\mathrm{PaO}_{2}$ partial pressure of oxygen in arterial blood, $\mathrm{PaCO}_{2}$ partial pressure of carbon dioxide in arterial blood, $\min -\mathrm{SpO}_{2}$ minimum oxygen saturation during 6-min walk test, BALF bronchoalveolar lavage fluid HLM hemosiderin-laden macrophage 
Table 5 Multivariate linear regression analysis for correlation with PVR

\begin{tabular}{llll}
\hline Characteristics & $\beta$ & $95 \% \mathrm{Cl}$ & $P$-value \\
\hline Six-minute walk distance & -0.391 & $-0.587 \sim-0.196$ & $<0.001$ \\
min-SpO $_{2}$ & -0.294 & $-0.455 \sim-0.133$ & 0.001 \\
HLM score & 0.265 & $0.088 \sim 0.412$ & 0.002 \\
& & & $R=0.595$
\end{tabular}

Definitions of abbreviations: PVR pulmonary vascular resistance, $\beta$ standardized partial regression coefficient, $95 \% \mathrm{Cl} 95 \%$ confidence interval, $\min -\mathrm{SpO}_{2}$ minimum oxygen saturation during 6-min walk test, HLM hemosiderin-laden macrophage

PVR, together with 6MWD and min- $\mathrm{SpO}_{2}$, which were evaluated by multivariate analysis.

Recently, several studies have reported that hemosiderin deposition or exaggerated numbers of HLM are observed in IPF $[3,4]$. Puxeddu et al. reported elevated HLM in BALF of patients with IPF [4]. By comparing BALF data from 47 radiologically or histologically proven IPF patients with data from healthy controls, they found significantly higher HLM in the IPF group regardless of smoking history, which is compatible with our findings.

HLM accumulation in IPF has been conjectured to be related to pulmonary hemodynamics in association with several pathological findings, including increased alveolar septal capillary density and microvessel density $[3,25]$. In the lung tissues of IPF patients, increased capillary density and angiogenesis were reported to exist in architecturally preserved lung areas next to the fibrotic areas [26-28]. Colombat et al. reported that the occlusion of venules and small pulmonary veins were observed in those architecturally preserved lung tissues in lung specimens from endstage IPF cases, which were associated with hemosiderin deposition in the interstitium and alveolar macrophages [25]. These findings suggest that occult bleeding in the lungs of patients with IPF is caused by the vulnerability of abnormal blood vessels and elevated blood pressure due to occlusion of those blood vessels.

In 2010, Kim et al. demonstrated that iron deposition in nonfibrotic lung areas from IPF were significant predictors of RVSP measured by echocardiography [3]. In a recent study, Puxeddu et al. reported that IPF patients with higher RVSP ( $>35 \mathrm{mmHg}$ ) evaluated by echocardiography showed significantly elevated HLM compared to those with RVSP $<35 \mathrm{mmHg}$. In the present study, the HLM scores of patients with IPF were significantly correlated with pulmonary hemodynamic parameters. We also evaluated mPAP and PVR by RHC, and the correlation of PVR with HLM score was confirmed using multivariate analysis. 6MWD $[11,12]$ and $\min -\mathrm{SpO}_{2}$ $[9,11]$ are well-known correlating factors for mPAP in patients with IPF. This study showed significant correlation between those factors and both mPAP (data not shown) and PVR, which is consistent with other reports on mPAP $[9,11,12]$.
Although the specific BAL cellular pattern of IPF is unclear, BAL may be performed if IPF is suspected, especially if other suspected conditions need to be excluded for differential diagnoses [26, 29]. Evaluation of the HLM score, which might be correlated with PVR, can provide additional information from BALF of patients with IPF for the detection of $\mathrm{PH}$ in the early phase of the disease, and prompt clinicians to perform further evaluation for $\mathrm{PH}$.

Several limitations of this study should be mentioned. First of all, this is a retrospective study in a single institute. Secondly, due to the high risk of performing BAL in patients with severe IPF, our cohort included patients with mainly mild to moderate IPF, and we cannot affirm that the results would be the same in all patients with IPF including severe cases. Nevertheless, our cohort showed a significant positive correlation between HLM score and PVR or mPAP. This fact suggests that hemosiderin deposition in the lungs of IPF is already significant even in mild to moderate IPF, and HLM score can reflect such early microvascular abnormality. Finally, pathological findings related with hemosiderin deposition were not reviewed for this study. Further pathological validation is needed to confirm our findings.

\section{Conclusions}

Among the data from patients with idiopathic pulmonary fibrosis, hemosiderin-laden macrophages in bronchoalveolarlavage fluid were significantly correlated with pulmonary hemodynamic parameters evaluated by right heart catheterization, and were an independent correlating factor of pulmonary vascular resistance.

\section{Abbreviations}

6MWD: 6-minute walk distance; 95\% Cl: 95\% confidence interval; BAL: Bronchoalveolar lavage; BALF: Bronchoalveolar lavage fluid;

$D_{L} C O$ : Diffusing capacity of the lung for carbon monoxide;

HLM: Hemosiderin-laden macrophages; IPF: Idiopathic pulmonary fibrosis; min- $\mathrm{SpO}_{2}$ : Minimum oxygen saturation during 6-minute walk test; mPAP: mean pulmonary arterial pressure; PVR: Pulmonary vascular resistance; RHC: Right heart catheterization; RVSP: Right ventricular systolic pressure; UIP: Usual interstitial pneumonia; $\beta$ : Standardized partial regression coefficient

\section{Acknowledgements}

This work was supported in part by a grant-in-aid for the respiratory failure research group from the Japanese Ministry of Health, Labor and Welfare, and the NPO Respiratory Disease Conference.

\section{Funding}

Not applicable.

\section{Availability of data and materials}

The dataset supporting the conclusions of this article is presented within the article. The detailed clinical data set is not publically available to protect research subject privacy and confidentiality.

\section{Authors' contributions}

JF and HT had full access to all the data in the study and takes responsibility for the integrity of the data and the accuracy of the data analysis. MA contributed to the statistical data analysis. YK and TF contributed to the design of the protocol. YK, TK, KK, KS and YH contributed to preparing the manuscript. TJ contributed to the interpretation of HRCT findings. Junya 
Fukuoka contributed to the interpretation of pathological findings from lung biopsy specimen. All authors read and approved the final manuscript.

\section{Competing interests}

The authors declare that they have no competing interests.

\section{Consent for publication}

Not applicable.

\section{Ethics approval and consent to participate}

This study was approved by Institutional Review Board of Tosei General Hospital (Review Board No. 480, Seto, Aichi, Japan). Informed consent has not been obtained from participants because this is a retrospective study and the data were analyzed anonymously.

\section{Author details}

'Department of Respiratory Medicine and Allergy, Tosei General Hospital, 160 Nishioiwake-cho, Seto-shi, Aichi 489-8642, Japan. ${ }^{2}$ Center of Advanced Medicine and Clinical Research, Nagoya University Hospital, 65 Tsurumai-cho, Showa-ku, Nagoya-shi, Aichi 466-0065, Japan. ${ }^{3}$ Department of Radiology, Kinki Central Hospital of Mutual Aid Association of Public School Teachers, 3-1 Kurumazuka, Itami-shi, Hyogo 664-8533, Japan. ${ }^{4}$ Department of Pathology, Nagasaki University Graduate School of Biomedical Sciences, 1-12-4 Sakamoto, Nagasaki-shi, Nagasaki, Japan. ${ }^{5}$ Department of Respiratory Medicine, Nagoya University Graduate School of Medicine, 65 Tsurumai-cho, Showa-ku, Nagoya-shi, Aichi 466-0065, Japan.

Received: 21 August 2016 Accepted: 28 January 2017

Published online: 06 February 2017

\section{References}

1. Golde DW, Drew WL, Klein HZ, Finley TN, Cline MJ. Occult pulmonary haemorrhage in leukaemia. Br Med J. 1975;2:166-8.

2. Kahn FW, Jones JM, England DM. Diagnosis of pulmonary hemorrhage in the immunocompromised host. Am Rev Respir Dis. 1987;136:155-60.

3. Kim KH, Maldonado F, Ryu JH, Eiken PW, Hartman TE, Bartholmai BJ, et al. Iron deposition and increased alveolar septal capillary density in nonfibrotic lung tissue are associated with pulmonary hypertension in idiopathic pulmonary fibrosis. Respir Res. 2010;11:37.

4. Puxeddu E, Comandini A, Cavalli F, Pezzuto G, D'Ambrosio C, Senis L, et al. Iron laden macrophages in idiopathic pulmonary fibrosis: The telltale of occult alveolar haemorrhage? Pulm Pharmacol Ther. 2014;28:35-40.

5. Raghu G, Collard HR, Egan JJ, Martinez FJ, Behr J, Brown KK, et al. An officia ATS/ERS/JRS/ALAT statement: idiopathic pulmonary fibrosis: evidence-based guidelines for diagnosis and management. Am J Respir Crit Care Med. 2011;183:788-824.

6. Rivera-Lebron BN, Forfia PR, Kreider M, Lee JC, Holmes JH, Kawut SM. Echocardiographic and hemodynamic predictors of mortality in idiopathic pulmonary fibrosis. Chest. 2013;144:564-70.

7. Corte TJ, Wort SJ, Gatzoulis MA, Macdonald P, Hansell DM, Wells AU. Pulmonary vascular resistance predicts early mortality in patients with diffuse fibrotic lung disease and suspected pulmonary hypertension. Thorax. 2009;64:883-8.

8. Nathan SD, Shlobin OA, Ahmad S, Urbanek S, Barnett SD. Pulmonary hypertension and pulmonary function testing in idiopathic pulmonary fibrosis. Chest. 2007;131:657-63.

9. Nathan SD, Shlobin OA, Barnett SD, Saggar R, Belperio JA, Ross DJ, et al. Right ventricular systolic pressure by echocardiography as a predictor of pulmonary hypertension in idiopathic pulmonary fibrosis. Respir Med. 2008;102:1305-10.

10. Zisman DA, Ross DJ, Belperio JA, Saggar R, Lynch 3rd JP, Ardehali A, et al. Prediction of pulmonary hypertension in idiopathic pulmonary fibrosis. Respir Med. 2007;101:2153-9.

11. Lettieri CJ, Nathan SD, Barnett SD, Ahmad S, Shorr AF. Prevalence and outcomes of pulmonary arterial hypertension in advanced idiopathic pulmonary fibrosis. Chest. 2006;129:746-52.

12. Modrykamien AM, Gudavalli R, McCarthy K, Parambil J. Echocardiography, 6-minute walk distance, and distance-saturation product as predictors of pulmonary arterial hypertension in idiopathic pulmonary fibrosis. Respir Care. 2010;55:584-8
13. Arnett FC, Edworthy SM, Bloch DA, McShane DJ, Fries JF, Cooper NS, et al. The American Rheumatism Association 1987 revised criteria for the classification of rheumatoid arthritis. Arthritis Rheum. 1988;31:315-24.

14. Tan EM, Cohen AS, Fries JF, Masi AT, McShane DJ, Rothfield NF, et al. The 1982 revised criteria for the classification of systemic lupus erythematosus. Arthritis Rheum. 1982;25:1271-7.

15. Hochberg MC. Updating the American College of Rheumatology revised criteria for the classification of systemic lupus erythematosus [letter]. Arthritis Rheum. 1997:40:1725.

16. Subcommittee for scleroderma criteria of the American Rheumatism Association Diagnostic and Therapeutic Criteria Committee. Preliminary criteria for the classification of systemic sclerosis (scleroderma). Arthritis Rheum. 1980:23:581-90.

17. Bohan A, Peter JB. Polymyositis and dermatomyositis (second of two parts). N Engl J Med. 1975:292:403-7.

18. Vitali C, Bombardieri S, Moutsopoulos HM, Balestrieri G, Bencivelli W, Bernstein RM, et al. Preliminary criteria for the classification of Sjögren's syndrome. Results of a prospective concerted action supported by the European Community. Arthritis Rheum. 1993;36:340-7.

19. Alarcon-Segovia D, Cardiel MH. Comparison between 3 diagnostic criteria for mixed connective tissue disease. Study of 593 patients. J Rheumatol. 1989;16:328-34.

20. Miller MR, Hankinson J, Brusasco V, Burgos F, Casaburi R, Coates A, et al. Standardisation of spirometry. Eur Respir J. 2005:26:319-38.

21. Macintyre N, Crapo RO, Viegi G, Johnson DC, van der Grinten CP, Brusasco V, et al. Standardisation of the single-breath determination of carbon monoxide uptake in the lung. Eur Respir J. 2005:26:720-35.

22. American Thoracic Society. ATS statement: guidelines for the six-minute walk test. Am J Respir Crit Care Med. 2002:166:111-7.

23. Meyer KC, Raghu G, Baughman RP, Brown KK, Costabel U, du Bois RM, et al. An official American Thoracic Society clinical practice guideline: the clinical utility of bronchoalveolar lavage cellular analysis in interstitial lung disease. Am J Respir Crit Care Med. 2012;185:1004-14.

24. De Lassence A, Fleury-Feith AJ, Escudier E, Beaune J, Bernaudin JF, Cordonnier C. Alveolar hemorrhage, diagnostic criteria and results in 194 immunocompromised hosts. Am J Respir Crit Care Med. 1995;151:157-63.

25. Colombat M, Mal H, Groussard O, Capron F, Thabut G, Jebrak G, et al. Pulmonary vascular lesions in end-stage idiopathic pulmonary fibrosis: histopathologic study on lung explant specimens and correlations with pulmonary hemodynamics. Hum Pathol. 2007;38:60-5.

26. Ebina M, Shimizukawa M, Shibata N, Kimura Y, Suzuki T, Endo M, et al. Heterogeneous increase in CD34-positive alveolar capillaries in idiopathic pulmonary fibrosis. Am J Respir Crit Care Med. 2004;169:1203-8.

27. Renzoni EA, Walsh DA, Salmon M, Wells AU, Sestini P, Nicholson AG, et al. Interstitial vascularity in fibrosing alveolitis. Am J Respir Crit Care Med. 2003; 167:438-43.

28. Magro CM, Waldman WJ, Knight DA, Allen JN, Nadasdy T, Frambach GE, et al. Idiopathic pulmonary fibrosis related to endothelial injury and antiendothelial cell antibodies. Hum Immunol. 2006;67:284-97.

29. Cottin V, Crestani B, Valeyre D, Wallaert B, Cadranel J, Dalphin JC, et al. Diagnosis and management of idiopathic pulmonary fibrosis: French practical guidelines. Eur Respir Rev. 2014;23:193-214.

\section{Submit your next manuscript to BioMed Central and we will help you at every step:}

- We accept pre-submission inquiries

- Our selector tool helps you to find the most relevant journal

- We provide round the clock customer support

- Convenient online submission

- Thorough peer review

- Inclusion in PubMed and all major indexing services

- Maximum visibility for your research

Submit your manuscript at www.biomedcentral.com/submit
C) Biomed Central 